\title{
Investigation of a Suspected Malaria Outbreak in Sokoto State, Nigeria, 2016
}

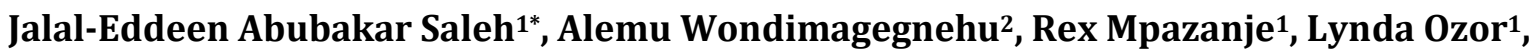 \\ Sule Abdullahi ${ }^{3}$ \\ ${ }^{1}$ Communicable Diseases, World Health Organization, Abuja, Nigeria \\ ${ }^{2}$ WR, World Health Organization Country Office, Abuja, Nigeria \\ ${ }^{3}$ Expanded Programme on Immunization, World Health Organization, Kano, Nigeria \\ Email: *salehj@who.int
}

How to cite this paper: Saleh, J.-E.A. Wondimagegnehu, A., Mpazanje, R., Ozor, L. and Abdullahi, S. (2017) Investigation of a Suspected Malaria Outbreak in Sokoto State, Nigeria, 2016. Open Access Library Journal, 4: e4246.

https://doi.org/10.4236/oalib.1104246

Received: December 11, 2017

Accepted: December 25, 2017

Published: December 28, 2017

Copyright (c) 2017 by authors and Open Access Library Inc.

This work is licensed under the Creative Commons Attribution International License (CC BY 4.0).

http://creativecommons.org/licenses/by/4.0/

\section{Open Access}

\begin{abstract}
Background: Malaria, a vector borne disease that contributes to the 17 percent of the global burden of infectious diseases, is preventable, treatable and completely curable. The disease is endemic in Nigeria, staggering at 27 percent prevalence and contributes up to 29 percent of global burden of the disease. It contributes to the high child mortality in Nigeria, attributed 30 percent of under five deaths. In October 2016, WHO team in Nigeria in collaboration with Nigerian Centre for Disease Control investigated a suspected malaria outbreak in Sokoto state of northwestern Nigeria. Materials and $\mathbf{M e}-$ thods: We reviewed hospital records of 190 patients who presented to the health facility with febrile illness, investigated and treated from 3/10/2016 to 25/10/2016. Data used during the study included age, sex, residential address, signs and symptoms. A cross-sectional survey was carried out in the region of the outbreak to assess the knowledge of the community on malaria control measures. SPSS version 24 was used for the data analyses. Results: Out of the 190 cases, 168 (88.4\%) tested positive for RDT and $22(11.5 \%)$ tested negative. The age range of the cases was from three months to 70 years (median: 14 years), the sex distribution was 118 (62.1\%) females and 72 (37.9\%) males, and the CFR of the RDT positive cases was $2.97 \%$ (5/168) with M:F ratio of 1:4. A survey to assess the knowledge of the affected community on malaria preventive measures shows 59.5\% (25/42) are aware of at least three out of four measures asked. Conclusion: Although government commitment to malaria control is commendable, this suspected outbreak has clearly brought to fore some gaps in the on-going malaria control in Sokoto state. Thus, there is a need for government to intensify health education programmes on environmental hygiene, state malaria control programme to strengthen awareness campaigns on malaria interventions as well as improve access to the available
\end{abstract}


interventions especially for the more vulnerable members of the community.

\section{Subject Areas}

Public Health

\section{Keywords}

Malaria, Outbreak Investigations, Sokoto, Nigeria

\section{Background}

Malaria is a vector-borne parasitic disease of Plasmodium origin transmitted by the infected female Anopheles mosquito. In Nigeria, though $A$. gambiae is the most dominant species across the country, $A$. arabiensis is most prevalent in the north and $A$. melas in the mangrove coastal zone of the country [1]. There are five different species of the Plasmodium parasites that cause malaria across the globe: $P$. falciparum, $P$. ovale, $P$. malariae, $P$. knowlesi, and $P$. vivax. Out of these species, $P$. falciparum is the most prevalent and virulent, and responsible for more than 95 percent of all malaria infections in Nigeria with $P$. malariae also isolated in children with mixed infections [1].

Globally, the incidence of malaria among population at risk has dropped by 21 percent [2]. Similarly, there is a significant global decline in malaria mortality by 62 percent between 2000 and 2015, and by 29 percent between 2010 and 2015 . Importantly, in children under the age of 5 years, the reduction is by 69 percent between 2000 and 2015 and by 35 percent between 2010 and 2015 [2]. It is on record that Nigeria and the Democratic Republic of Congo (DRC) are considered the epicenters of malaria transmission contributing up to 40 percent of the global malaria burden [3]. However, Nigeria which contributes about $29 \%$ of the over $80 \%$ global burden of malaria has recorded a significant improvement within the last decade [3]. Evidence from a national survey showed a decline in malaria prevalence from $42 \%$ in 2010 to $27 \%$ in 2015 , and in the northwestern zone of the country, the prevalence has dropped from 48 percent to 37 percent between 2010 and 2015 [1]. Sokoto state, northwestern Nigeria, the epicenter of the outbreak, has a prevalence of 46.6 percent ranking third out of the 36 states including Abuja in the country [1].

Although many countries across the globe have significantly reduced the burden of malaria with the recommended core prevention tools, there still exist major gaps in coverage especially in the low-income countries [2] [4] [5]. In the sub-Saharan African region, $43 \%$ of people that are at risk of malaria are not protected from mosquito bites with the LLINs or indoor spraying of insecticides and $69 \%$ of pregnant women lack access to the recommended minimum three doses of intermittent preventive treatment in pregnancy (IPTp) during antenatal visits [2] [4] [5].

In the sub-Saharan Africa, 663 million cases of malaria were prevented be- 
tween 2010 and 2015 as a direct result of the scale-up core malaria interventions with insecticide-treated nets having the greatest impact averting about $69 \%$ of cases of malaria [2] [4] [5]. Thus, for country programmes to achieve malaria elimination, there is a need for adherence to the key preventive measures against malaria which include the use of long-lasting insecticide-treated nets (LLINs), indoor residual spraying (IRS), use of preventive medicines in the most vulnerable groups (IPTp, IPTi and SMC) and improving access to these critical tools [6].

\section{Method}

Following a report from the Sokoto state malaria elimination unit on the $21^{\text {st }}$ October 2016 that there was an unusual increase in the number of malaria cases that tested RDT positive in October 2016 from three health facilities; PHC Kofar Kade, Assada PHC and PHC Kofar Rini from Sarkin Adar Gandu ward of Sokoto north local government area of Sokoto state, a line-list of 190 suspected malaria patients who presented with fever, investigated and treated between 1/10/2016-30/10/2016 was prepared by the surveillance officer at the World Health Organization Sokoto state, northwest Nigeria. The case data for RDT positive cases were obtained and compared with same period in the preceding year which revealed an upsurge in the number of malaria cases based on the positive RDT tests in the period under review. Although the period of investigation coincided with a period of high malaria transmission across the northwest zone, the upsurge in cases seen as compared with the same period the preceding year did not reach an outbreak threshold. The data collected included age, sex, residential address, signs, and symptoms, RDT status, treatment and outcome.

In accordance with the WHO guidelines, all febrile illnesses that tested positive using rapid diagnostic tool (RDT) or microscopy were considered as confirmed malaria and treatment given using artemisinin combination drugs (ACTs). However, the few cases that presented with epistaxis or tested negative for RDTs were referred to a secondary health centre for further management. Additionally, a survey involving 42 caregivers was conducted in the affected communities to assess their knowledge about malaria prevention measures. The selection criteria for the households to interview the caregivers were through a stratified random sampling. The data were collated and analysed using SPSS version 24 .

\section{Result}

The outbreak started on Epi week 40 (43/190), peaked on week 41 (71/190), started dropping in week $42(68 / 190)$ and levelled off in week 43 (8/190) (Figure 1). The Epi weeks during which deaths were recorded are weeks 40 (2), 41 (1), and 42 (2). Figure 2 shows gender distribution of cases 118 (62.1\%) females and $72(37.9 \%)$ males, and a median age of 14 years (Table 1$)$. There was no missing data recorded. 


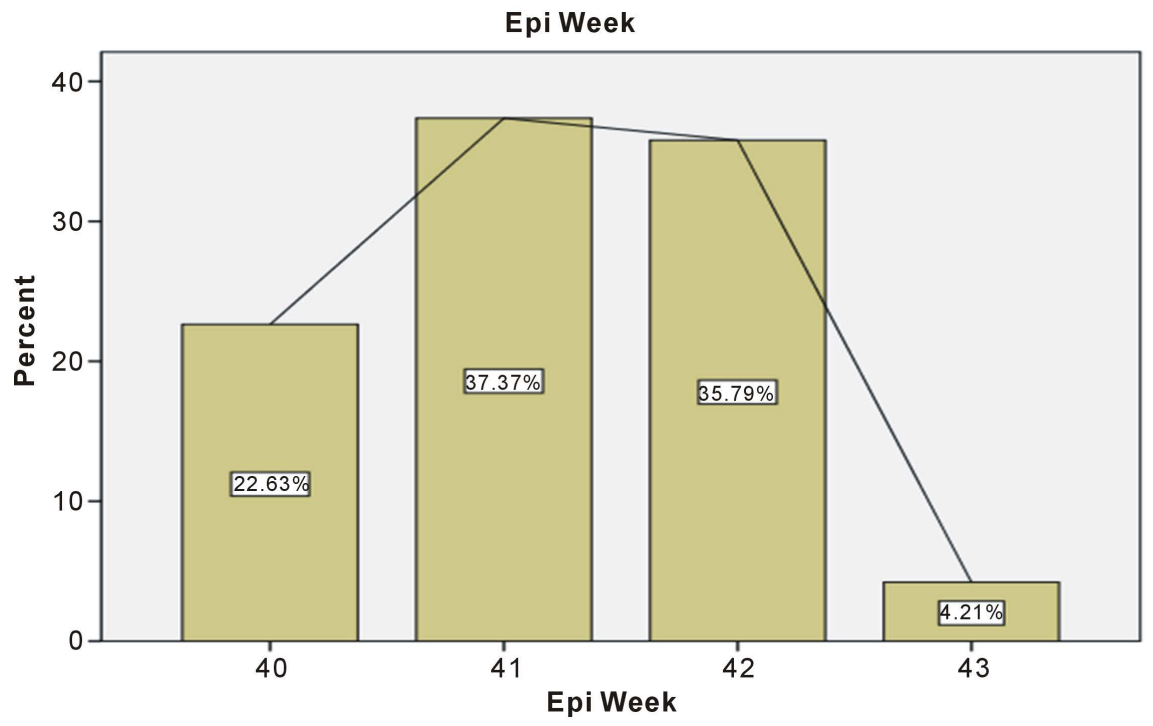

Figure 1. Epidemiological week of cases.

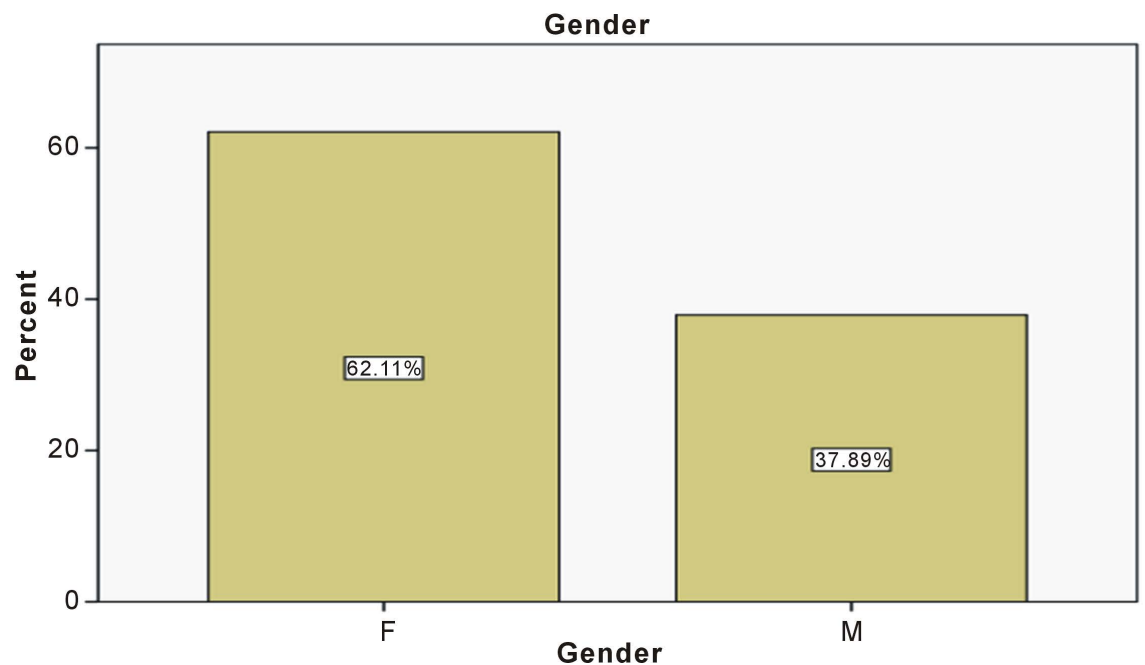

Figure 2. Gender distribution.

Table 1. Descriptive statistics.

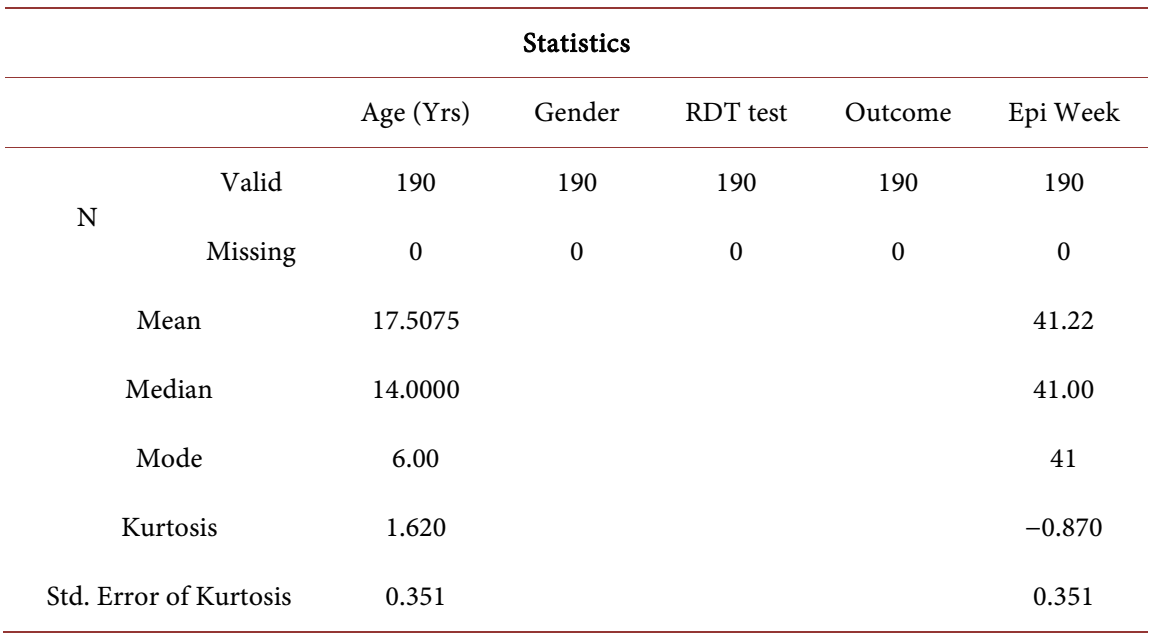


The clinical presentation of the patients was high-grade fever, severe headaches, watery diarrhoea, vomiting and mild anaemia in the paediatric age group. While there was no reported jaundice, bleeding from the gums or petechial haemorrhages, $1.6 \%(3 / 190)$ of the patients presented with epistaxis. As shown in Figure 3 there were 168 (88.4\%) who tested positive for RDT and 22 (11.6\%) tested negative. The RDT negative cases were given symptomatic treatment and referred to secondary health facilities for further management. Figure 4 shows the 190 cases who presented to the health facilities-survived 163 (85.8\%), referred $22(11.6 \%)$ and died $5(2.6 \%)$. Thus, the CFR was $2.6 \%$ with M:F ratio of 1:4. Furthermore, the outcome of the cross-sectional survey conducted in the affected community to assess their knowledge on malaria preventive measures reveals 59.5\% (25/42) are aware of at least three out of four measures that they were asked and $40.5 \%$ (17/42) fail short of that (Figure 5).

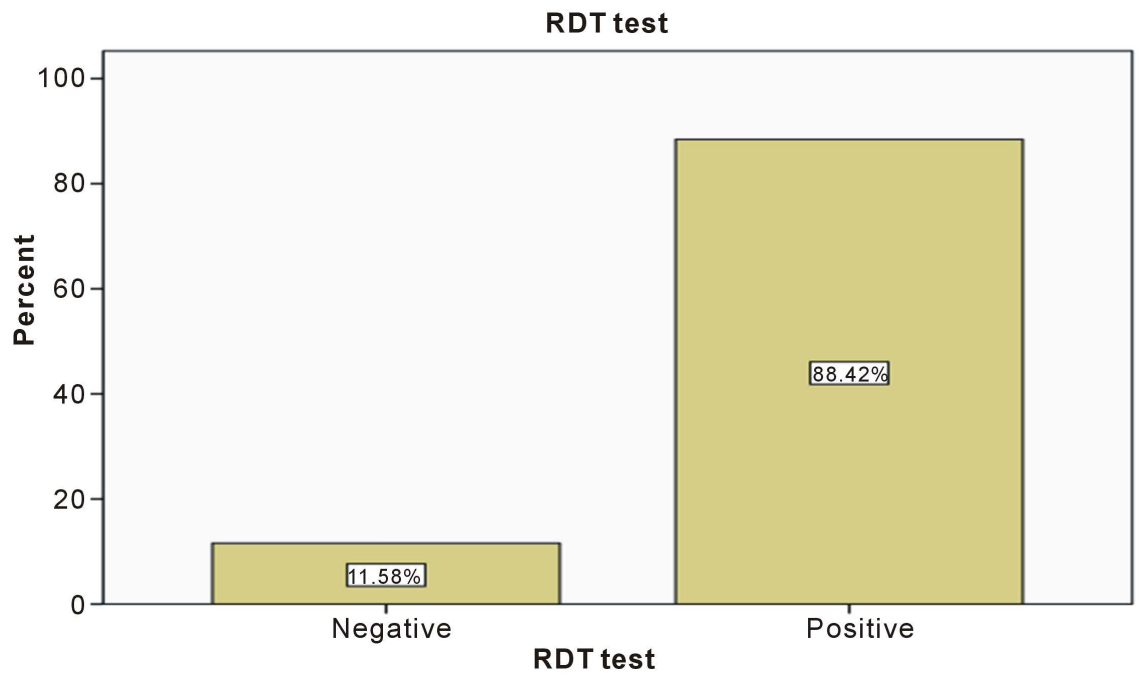

Figure 3. RDT test results.

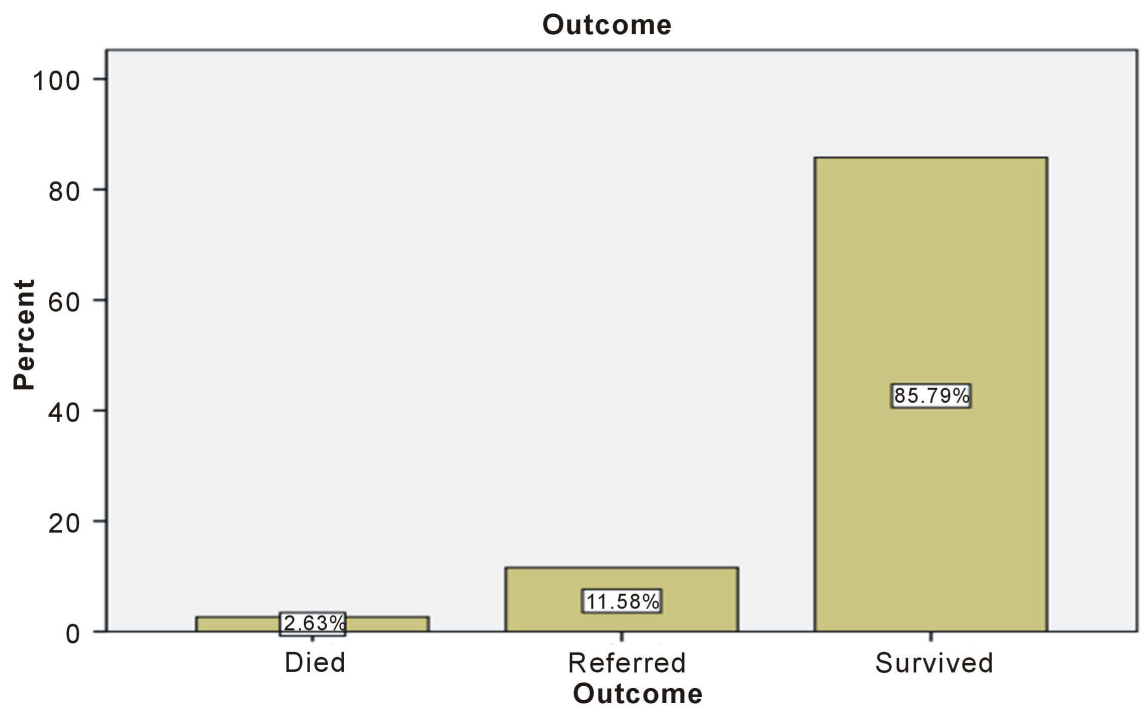

Figure 4. Outcome of the malaria outbreak cases. 


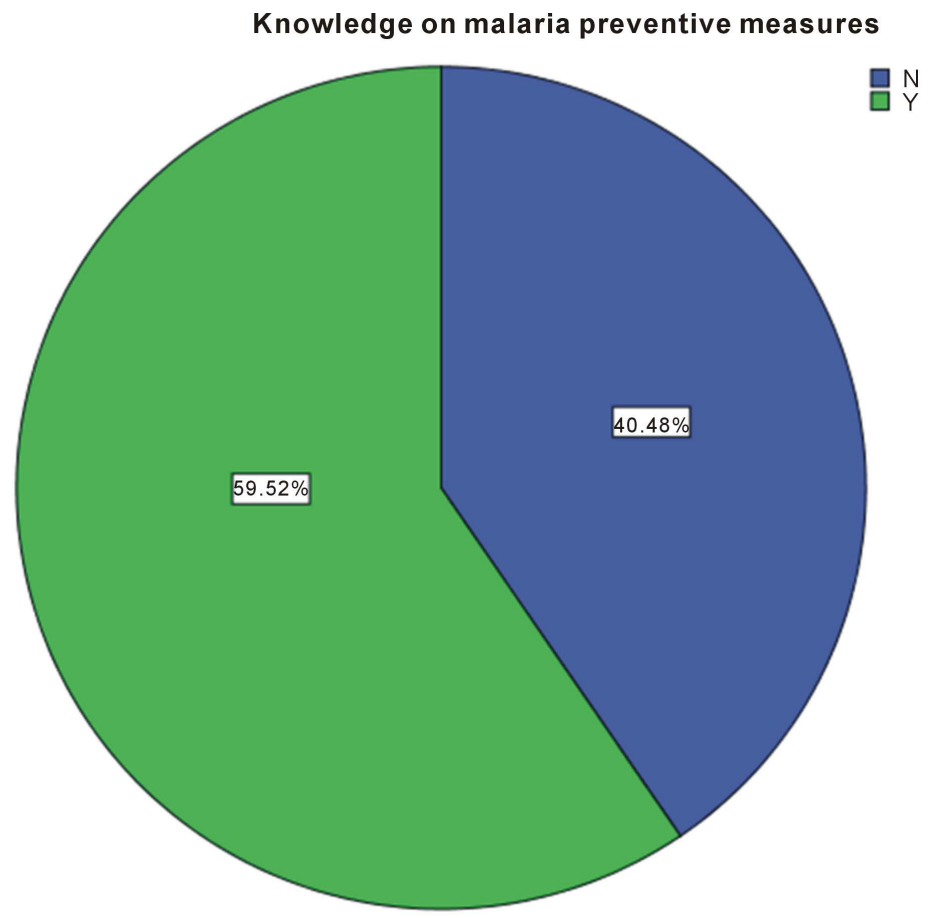

Figure 5. Knowledge of respondents on at least three of four measures of malaria prevention.

\section{Discussion}

Sokoto state ranks third in terms of malaria prevalence (46.6\%) in a zone that has the highest prevalence of malaria (37.10\%) in the country [1]; the commitment of the government and donor communities toward malaria control is profound. Though malaria vector control strategies include Indoor residual spraying and larval source management, LLINs ownership is by far the most prevalent. Incidentally, the northwest zone also has the highest ownership of LLINs (36.7\%) when compared with the other five geo-political zones in the country [1]. However, only $24 \%$ of households in Sokoto state have at least 1 net for every 2 persons.

While it is part of government policy that pregnant women should be given LLINs and IPTp during routine antenatal visits and children under the age of 1 year to get LLIN on completion of routine immunizations [7], often there is stock out of the LLINs in most of the health facilities.

Sokoto North local government area of Sokoto state is known to lack adequate environmental hygiene leaving stagnant waters as breeding sites for the mosquitoes. There is inadequate coverage with malaria prevention strategies including seasonal malaria chemoprevention and Intermittent Preventive Therapy in Pregnancy, which ought to prevent malaria or its severe forms in children and pregnant women who are hitherto exposed to mosquito bites. The inadequate distribution and use of LLINs by especially those most vulnerable in the community, and poor access to adequate malaria diagnosis and quality ACTs result in patients buying expired and/or ineffective ACTs from unlicensed medicine 
vendors.

There is a need to underscore the point that the ward of the malaria outbreak has some interesting public health issues, some of which include the presence of the largest cattle market in the state with a large number of traders coming from the neighboring states and countries weekly; it hosts the central market and $\mathrm{Ab}$ attoir that serves the state, and in December 2015 a compatible polio virus was picked from a child. Furthermore, the survey has revealed that about half of care-givers in the affected community lack adequate knowledge of malaria and the available preventive measures.

The recommendations include the need for state government through the state malaria control programme to strengthen awareness campaigns to educate the populace on malaria and on the available interventions especially those at the hard-to-reach areas, to strengthen health education programmes on environmental hygiene, to improve access to the available interventions to especially the more vulnerable ones, to establish secondary health facility to complement the only existing primary health facility, and to check out the activities of PMVs on sale of expired ineffective ACTs to the community [2] [4] [6] [7] [8] [9].

\section{Limitations}

This study is limited by the fact that those interviewed to assess their knowledge on malaria preventive measures in the affected ward were not necessarily the patients who received treatment at the health facilities during the outbreak.

\section{Declarations/Competing Interests}

We declare that there is no any potential conflict of interest or royalty associated with the manuscript.

We declare that no funding was received from any source for this outbreak investigation.

\section{Acknowledgements}

The outbreak investigation was supported by the World Health Organization as part of its Technical Assistance to malaria control and elimination in Nigeria.

We acknowledge with gratitude the cooperation of officials from the Sokoto $\mathrm{SMoH}$.

\section{References}

[1] NMEP, NPopC, NBS, and ICF International (2016) Nigeria Malaria Indicator Survey 2015. NMEP, NPopC, and ICF International, Abuja, Nigeria, and Rockville, Maryland, USA.

[2] World Health Organization (2016) WHO Global Malaria Programme, World Malaria Report 2016. WHO Document Production Service, Geneva.

[3] World Health Organization (2014) WHO Global Malaria Programme, World Malaria Report 2014. WHO Document Production Service, Geneva. http://www.who.int/malaria/publications/world-malaria-report-2016/en/ 
[4] WHO Global Malaria Programme (2016). http://www.who.int/malaria/en/

[5] WHO (2017) World Malaria Day.

http://www.who.int/campaigns/malaria-day/2017/en/

[6] GTS (2016) Global Technical Strategy for Malaria 2016-2030. http://www.who.int/malaria/publications/atoz/9789241564991/en/

[7] NMEP (2014) National Malaria Strategic Plan 2014-2020. 2014. NMEP and Roll Back Malaria, Abuja.

[8] Sharma, R. (2006) Epidemiological Investigation of Malaria Outbreak in Village Sanjet, District Gandhi Nagar (Gujarat). Indian Journal of Preventive and Social Medicine, 37, 125-132.

[9] Salihu, O.M. and Sanni, N.A. (2013) Malaria Burden and the Effectiveness of Malaria Control Measures in Nigeria: A Case Study of Asa Local Government Area of Kwara State. Journal of Economics and Sustainable Development, 4, Online. http://iiste.org/Journals/index.php/JEDS/article/view/4590 